\title{
CONTROL OF NETWORKED SYSTEMS CONTAINING MULTIPLE AGENTS
}

\author{
Jose B. Cruz, Jr. ${ }^{1,2}$, Gregory Tangonan ${ }^{2}$, Raymond R. Tan ${ }^{3}$, \\ ${ }^{I}$ The Ohio State University, Columbus USA; \\ ${ }^{2}$ Ateneo De Manila University, Manila Philippines ${ }^{3}$ De La Salle University,Manila, Philippines; \\ jbcruz@ieee.orgraymond.tan@dlsu.edu.ph goriot@mac.com \\ Nathaniel Libatique ${ }^{2}$, Fabian M. Dayrit ${ }^{2}$, Alvin B. Culaba ${ }^{3}$ \\ ${ }^{2}$ Ateneo De Manila University, Manila Philippines; ${ }^{3}$ De La Salle University,Manila, Philippines; \\ nlibatique@ateneo.edu.fdavrit@ateneo.edu alvin.culaba@dlsu.edu.ph
}

Keywords: Multi-agent systems, game theory, complex systems, networked control systems.

\begin{abstract}
This position paper illustrates the use of a natural framework for the modelling, analysis, and design of engineering systems that involve two or more controllers, each of which has an associated objective function. Such systems arise when ordinary single controller systems are networked through communication links so that the information available to each controller may contain aspects of the other systems' states and the optimization of each objective function is no longer decoupled from each other. Single controller optimization is no longer directly applicable. Appropriate to the study of such systems is the theory of games that has been developing in mathematics, economics, and engineering for the past 60 years. There are extensive applications in economics, but in engineering the applications are scarce. In recent years, there has been great attention to global problems such as the negative environmental impact of energy use, and global warming. These problems arise from complex systems with multiple controllers. Among the approaches for dealing with the problems, there should be one on a total systems approach with a game theory base. A natural framework for this is the subject of this policy paper.
\end{abstract}

\section{INTRODUCTION}

In this position paper we establish the benefits and advantages of explicitly including multiple agents in the modelling and control of networked engineering systems, when in fact, multiple agents are present in the application systems. The agents are not necessarily cooperating in a team and not necessarily antagonistic against each other, although in some applications they might be cooperating as a team. Some global problems with significant technological components are (a) integration of renewable energy sources (such as solar, geothermal, wind, hydro, and biological) with the traditional fossil energy sources, to reduce negative impact on the environment, (b) recycling of wastewater to produce clean water, to conserve scarce fresh water resources, (c) mitigating damages due to disasters such as typhoons, hurricanes, floods, and earthquakes. These national and international problems are also examples of complex systems. Complexity arises because of large numbers of smaller systems that are networked together, and total system behaviour is not easily inferred from individual behaviours of the component systems. These complex systems are characterized by the presence of many stakeholders, starting from the national government, to provincial/state governments, private enterprises of suppliers, industry associations, and large blocks of consumers. The stakeholders generally have policies that translate to actions affecting the system. 
Notwithstanding announced plans to the contrary, the complex systems typically evolve piece-meal, and unexpected and undesirable effects are addressed piece-meal. Finally, complex systems have numerous time lags throughout and stability is a crucial issue that could lead to a total collapse if not addressed properly.

A specific scenario for a networked system is the following: we can project a boom in ethanol production in some countries, even exporting of ethanol (after meeting local needs for ethanol), using sugar cane. For the same country can also project a return to the export of sugar as well. The sugar industry is terribly inefficient and unkind to labour six months of work and down for 6 months. The ethanol industry needs sugar cane 12 months of the year. The options for farmers have just changed what is their way of optimizing their land and/or labour? The government needs to figure out how to provide a good price of sugar for consumers, to protect the growing ethanol market, and to encourage investment by distillers moving into the ethanol industry through incentives such as tax breaks. This ecosystem is extremely rich in control problems to consider. Various stakeholders or agents need to understand various solution concepts and different perspectives. A decision support system that reflects the interactions of all the stakeholders in the modelling and control strategies would be highly useful.

The presence of multiple stakeholders in complex systems can be studied through mathematical game theory. Much of the literature is for static systems with very few exceptions (Nash 1950, Nash 1951, von Neumann and Morganstern 1947, von Stackelberg 1952). Dynamics can be studied using standard methods in mathematics and engineering. Yet, there are no general computerbased decision aids as tools for the design and analysis of dynamic complex systems. To be sure the challenge is not trivial and it requires significant research effort.

The bulk of control theory pertains to dynamic systems with a single controller (Bellman 1957, Pontryagin, Boltyanskii, Gamkrelidze, Mischenko 1962). With the emergence of networked control systems (Wang and Liu 2008) whereby previously separate individual subsystems with their individual controls are linked through communication networks, the information available at each subsystem through the links may generally contain aspects of the other systems. If a subsystem controller were to optimize a performance criterion associated with that subsystem, the performance criterion may contain variables pertaining to the other subsystems because of the network links and a dilemma of how to proceed is encountered.

There have been design approaches that ignore the presence of the links in the network. Subsequently the systems are analyzed to check robustness against the neglected connections. In the case of two interconnected control systems, worst case scenarios have been assumed in the design, in the sense that the controller of the other subsystem is assumed to make the performance criterion of the system as bad as possible while the controller of the system maximizes the performance of the resulting worst case. The theory of maxmin (or minmax) optimization is applied. In many applications the performance criteria of the two control systems are not opposite of each other, so that the minmax design is overly conservative. Furthermore, when the system is dynamic and the controls involve feedback, the structure of the feedback control of the other system needs to be known, in order to perform a correct minmax optimization with the correct total system dynamics. Thus the minmax approach could be problematic unless the other system including its feedback structure is modelled properly.

When the agents are cooperating, the individual performance criteria may be combined as a single convex linear combination using the Pareto-optimal concept, and once again the theory for single controller systems may be used. In applications, there is the additional task of choosing the weights in the convex linear combination. A special case arises when the performance criteria are identical and the choice of weights is immaterial. This is the team-optimal problem.

Unlike engineering systems, economic systems are modelled, analyzed, and optimized using multiple agents. In fact in the ideal case, there is an infinite number of consumers and an infinite number of suppliers interacting in a market. However, the bulk of the literature is for static systems with very few exceptions. The bulk of the literature in the field of operations research with respect to multiple agents is likewise for static systems.

\section{NETWORKED STATIC CONTROL SYTEMS}

To focus on the effects of multiple agents in a networked control system, let us initially consider static systems to eliminate one dimension of complexity induced by dynamics. 
Furthermore, even the static area could be utilized to great advantage in dealing with large complex systems. Suppose we have two networked control systems of producers of renewable energy. The productions are modelled by

$$
\begin{aligned}
& x_{1}=f_{1}\left(u_{1}\right) \\
& x_{2}=f_{2}\left(u_{2}\right)
\end{aligned}
$$

where $x_{1}$ and $x_{2}$ are quantities of renewable energy produced by Companies 1 and 2 respectively, and $u_{1}$ and $u_{2}$ are resources (controls) used to produce $x_{1}$ and $x_{2}$ respectively. The functions $f_{1}$ and $f_{2}$ are monotonically increasing so their unique inverses exist. The renewable energy products are sold in a market and the price $P$ is determined from a supply curve that relates price to total demand $X$

$$
P=m_{1} X+b_{1}
$$

and the total demand $\mathrm{X}$ is equal to the total supply

$$
X=x_{1}+x_{2}
$$

The parameters $m_{1}$ and $b_{1}$ are given and $m_{1}>0$, $b_{1}<0$. The costs for producing the renewable energy products are

$$
\begin{aligned}
& \mathrm{C} 1=g_{1}\left(x_{1}\right) \\
& \mathrm{C} 2=g_{2}\left(x_{2}\right)
\end{aligned}
$$

where $g_{1}$ and $g_{2}$ are nonlinear functions. Each company wants to maximize its profit, which is revenue minus cost. For Company 1 the profit is

$$
\begin{aligned}
& \text { Profit } 1=P x_{1}-g_{1}\left(x_{1}\right) \\
& \text { Profit } 2=P x_{2}-g_{2}\left(x_{2}\right)
\end{aligned}
$$

It is not a simple matter for Company 1 to maximize its profit Profit1 with respect to $x_{1}$ because $P$ in Profit1 contains $x_{2}$, which is not under its control. Similarly for Company 2 , it is not a simple matter to maximize Profit 2 with respect to $x_{2}$ because $P$ contains $x_{1}$.

This illustrates the intrinsic difference between a single controller problem and a problem with multiple controllers or multiple agents, such as the example above where the two static control systems are networked through the market mechanism where their outputs are sold. From the point of view of single controller theory, for example in the design of $u_{1}$, Company 1 may simply assume a value for $u_{2}$ or $x_{2}$ and proceed to maximize Profitl with respect to $x_{1}$. Except for some very lucky choice of $u_{2}$ by Company 1 , when Company 2 chooses $u_{2}$ using an assumed value of $u_{1}$, Company 2 will obtain a value of $u_{2}$ different from the one assumed by Company 1 , posing a dilemma for both companies.

Next let us consider each company's worst-case design whereby Company 1 assumes that Company 2 chooses u2 to minimize Profit1. Then Company 1 maximizes Profit1 resulting in its maxmin (or worstcase) design. Similarly Company 2 may proceed to calculate its worst-case design. When both apply their worst-case designs, their resulting profits will be generally higher but in any case no worse than the worst-case profits they previously calculated. The pair of separately calculated worst-case controls will generally not lead to the worst case for either company. Still, in general their designs would be conservative because the companies are not trying to destroy each other by making each other's profit as small as possible.

In the theory of games that applies to systems with multiple agents, there are many solution concepts that go beyond single controller optimization. For example, one may consider the Nash equilibrium concept (Nash 1950, Nash 1951) whereby when $\left(u_{1} N, u_{2} N\right)$ is a Nash solution pair and Company 1 chooses a control $u 1$ that is different from $u_{1} N$, but Company 2 still uses $u_{2} N$, the resulting profit of Company 1 can not be higher than that when both use their Nash controls. There is also a Stackelberg (von Stackelberg 1952) or LeaderFollower solution concept whereby one subsystem controller is dominant or more powerful than the others. The leader's control is announced in advance and all other controllers know what the leader's control is before they choose their own controls. In the case of Stackelberg games, it is of particular interest to determine the role of the dominant player in inducing desirable behaviour from low-level players through incentives or disincentives. The implications of such mechanisms are clearly evident for situations in which, for example, it is desired to determine government policies to facilitate environmentally beneficial behaviour from the private sector (e.g., Aviso et al 2010). The Stackelberg hierarchy may have more than two levels.

\section{NETWORKED DYNAMIC CONTROL SYSTEMS}

A system that is more general than the class considered in Section 2 is one where the individual control systems are dynamic. If the systems are 
discrete-time the individual models may be of the form

$$
\left.x_{i}(k+1)\right)=f_{i}\left(x_{i}(k), u_{i}(k), k\right)
$$

$i=1, \ldots, N, k=0, \ldots, M$, where $x_{i}$ is the state vector of system $i$, with dimension $n_{1} ; u_{i}$ is the control vector of system $i$, with dimension $m_{i}$, and $k$ is discrete time with integer values from 0 to $M$. The vector functions $f_{i}$ are mappings from the spaces of the arguments to the space of $x_{i}$ and $n_{i}$, $m_{i}, N$ and $M$ are given. The network connections may be modelled by an algebraic equation

$$
G\left(x_{1}, \ldots, x_{n}\right)=0
$$

where $G$ may be a scalar or vector of a given dimension. Associated with each system $i$, may be a scalar performance index or cost function

$$
J_{i}=C_{i}\left(x_{i}(M)\right)+\sum_{j=0}^{M-1} L_{i}\left(x_{i}(j), u_{i}(j)\right)
$$

If $J_{i}$ represents total cost for the entire horizon from 0 to $\mathrm{M}$, then $C_{i}\left(x_{i}(M)\right)$ represents the incremental cost at the terminal time, and $L_{i}\left(x_{i}(j), u_{i}(j)\right)$ represents the incremental cost during time $j$. As in the static case, there will be a dilemma in a simple dynamic optimization of $J_{i}$, in Equation (11) with respect to the control vector $u_{i}(j)$ sequence because the control may be in feedback form and even if only $x_{i}(j)$ is used for feedback at time $j, x_{i}$ is not independent of the other system states because of the network coupling modelled by Equation (10).

A more general effect of the network connection represented by Equation (10) may be a change in the individual control system model from Equation (9) to Equation (12)

$x_{i}(k+1)=f_{i}\left(x_{1}(k), \ldots x_{N}(k), u_{i}(k)\right.$

$i=1, \ldots, N, k=0, \ldots, M$

and the constraint represented by Equation (10) may remain.

For systems that are modelled as continuoustime processes a typical description in state variable form is given by the vector differential equation

$$
d x_{i}(t) / d t=f_{i}\left(x_{1}(t), \ldots, x_{N}(t), u_{i}(t)\right)
$$

where $x_{i}$ is the state vector of system $i$, with dimension $n_{1} ; u_{i}$ is the control vector of system $i$, with dimension $m_{i}$, and $\mathrm{t}$ is continuous time with values in the interval $[0, \mathrm{~T}]$, and $\mathrm{T}$ is a specified real number. Instead of the cost function in Equation (11) the continuous time version is an integral analogous to the sum in Equation (11)

$$
J_{i}=\phi_{f}\left(x_{i}(T)\right)+\int_{0}^{T} L_{i}\left(x_{i}(t), u_{i}(t), \mathrm{t}\right) \mathrm{dt}
$$

Because of the links in the network there may be a constraint as in Equation (10). The standard dynamic optimization of the integral cost functional with respect to the vector functions $\left.u_{i}(t)\right)$ for $t$ in the interval $[0, T]$ poses a dilemma because the functional may depend on the states of the other subsystems through the constraint in Equation (10).

In general, the direct application of dynamic optimization for single controllers becomes a problematic issue. The field of dynamic game theory offers potential benefits in the design and analysis of such systems (Isaacs 1955, Basar and Cruz 1982, Basar and Olsder 1998, Starr and Ho 1969a, Starr and Ho 1969b, Chen and Cruz 1972, Simaan and Cruz 1973a-c, Cruz 1975, Castanon and Athans 1976). Macroeconomics has completely adopted concepts from dynamic game theory. For multiple agent dynamic engineering systems the application of multi-agent models and equilibrium theories of dynamic games would be beneficial also.

\section{ILLUSTRATIVE EXAMPLE}

In this section we consider a single, simplified composite energy system with only one state variable $\left(\mathrm{x}_{t}\right)$ but two decision-makers, each with a control variable. We consider only finite states of zero, 1 , and 2 and finite controls 0 and 1 , and two time stages. For each controller there will be an associated incremental cost at each time stage and a total cost for the two time stages. We will analyze the network using some of the solution concepts in dynamic game theory.

Table 1. State Transitions in Period 1 (Simaan and Cruz 1973b)

\begin{tabular}{|c|c|c|c|c|}
\hline \multirow{2}{*}{} & \multicolumn{4}{|c|}{ Controllers' Decisions } \\
\cline { 2 - 5 } & $(0,0)$ & $(0,1)$ & $(1,0)$ & $(1,1)$ \\
\hline $\mathrm{x}_{0}=1$ & $\mathrm{x}_{1}=1$ & $\mathrm{x}_{1}=2$ & $\mathrm{x}_{1}=0$ & $\mathrm{x}_{1}=1$ \\
\hline
\end{tabular}

Controller 1 is assumed to be the upstream agricultural sector of a biofuel supply chain, similar to that considered in Cruz, Tan, Culaba, and Balacillo 2009, while Controller 2 is the downstream 
sector comprised of the biofuel processing sector. In each time period, each controller is faced with the option of expanding $(u=0)$ or maintaining $(u=1)$ current production capacity. The composite system is described by a trivalent state variable which indicates upstream (agricultural) deficit $(x=0)$, balanced production $(\mathrm{x}=1)$, or upstream surplus ( $\mathrm{x}$ $=2$ ). This is a biofuel supply chain interpretation of the numerical example that appeared in Starr and Ho 1969 a,b and Simaan and Cruz 1973a,b. Tables 1 and 2, which are based on the game described in Simaan and Cruz 1973b, show the possible state transitions arising from decisions in this stylized energy system. As each controller is faced with a binary decision in a given time period, over the entire planning horizon each will have four possible decisions, namely, $(0,0),(0,1),(1,0)$ and $(1,1)$. For open-loop control structure, i.e., the controls are functions of time (stage) only, each of the two controllers have four possible decision sequences, and the two-stage game may be expressed in bimatrix form as in Table 3, wherein the first and second entries are the cumulative costs borne by the two controllers over the entire time horizon. See Simaan and Cruz 1973 b for the incremental costs.

Table 2. State Transitions in Period 2 (Simaan and Cruz 1973b)

\begin{tabular}{|c|c|c|c|c|}
\hline \multirow{2}{*}{} & \multicolumn{4}{|c|}{ Controllers' Decisions } \\
\cline { 2 - 5 } & $(0,0)$ & $(0,1)$ & $(1,0)$ & $(1,1)$ \\
\hline $\mathrm{x}_{1}=0$ & $\mathrm{x}_{2}=2$ & $\mathrm{x}_{2}=1$ & $\mathrm{x}_{2}=0$ & $\mathrm{x}_{2}=0$ \\
\hline $\mathrm{x}_{1}=1$ & $\mathrm{x}_{2}=1$ & $\mathrm{x}_{2}=2$ & $\mathrm{x}_{2}=0$ & $\mathrm{x}_{2}=1$ \\
\hline $\mathrm{x}_{1}=2$ & $\mathrm{x}_{2}=2$ & $\mathrm{x}_{2}=2$ & $\mathrm{x}_{2}=1$ & $\mathrm{x}_{2}=0$ \\
\hline
\end{tabular}

Table 3. Cumulative Cost for Open-Loop Bi-Matrix Game

(Simaan and Cruz, 1973b)

\begin{tabular}{|c|c|c|c|c|c|}
\hline & \multicolumn{4}{|c|}{ Controller 2} \\
\hline & & $(0,0)$ & $(0,1)$ & $(1,0)$ & $(1,1)$ \\
\hline \multirow{4}{*}{$\begin{array}{l}\overline{\overline{0}} \\
\overline{\overline{0}} \\
\stackrel{0}{0} \\
0\end{array}$} & $(0,0)$ & 8,8 & $11,6^{* *}$ & $10,-2$ & 11,0 \\
\hline & $(0,1)$ & 6,4 & 12,3 & 7,2 & 12,4 \\
\hline & $(1,0)$ & 5,12 & 20,15 & 5,11 & $8,9 *$ \\
\hline & $(1,1)$ & $6,5^{* * *}$ & 16,7 & 3,7 & 9,6 \\
\hline \multicolumn{6}{|c|}{ *Nash equilibrium } \\
\hline \multicolumn{6}{|c|}{$\begin{array}{l}* * \text { Open-loop Stackelberg equilibrium with Controller } 2 \\
\text { as leader }\end{array}$} \\
\hline \multicolumn{6}{|c|}{$\begin{array}{l}* * * \text { Open-loop Stackelberg equilibrium with Controller } \\
1 \text { as leader }\end{array}$} \\
\hline
\end{tabular}

If we assume that neither the upstream nor downstream sectors of the energy supply chain dominate the game, the system naturally tends toward the Nash equilibrium as indicated in Table 3. In this case, each decision maker identifies his rational reaction, which is the response that minimizes his cost for any possible action by the other player. The Nash equilibrium is the intersection of the rational reactions of the two decision makers. They both commit to an open-loop sequence at the beginning of the horizon. The upstream sector maintains production capacity in the first time period, and expands production in the second period, while the downstream sector maintains its capacity throughout. As a result, the energy system is at a state of surplus farm production at the end of the time horizon analyzed. Note that this state is reached without any centralized direction, and emerges purely from the self-interested action of the two agents.

The energy system evolves differently if either sector were dominant. For instance, if the downstream (fuel processing) sector acted as the leader, the system reaches the open-loop Stackelberg equilibrium indicated in Table 3 . In this scenario, the dominant decision-maker selects his action so as to minimize his cost, having anticipated that the follower's response is the latter's rational reaction as in the Nash case. It would commit in advance that it would increase production capacity in the first period, but maintain it in the second period. The agricultural sector, the follower, would increase production in both periods. The energy supply chain thus also reaches a state of surplus upstream production capacity (i.e., excess biofuel feedstock) as in the Nash game, except that the cost burden of the farmers would have increased while those of the processors would have gone down. Note that the leader's Stackelberg solution can be no worse than his corresponding Nash solution (Simaan and Cruz, 1973a,b).

A similar analysis can be made for the case wherein the upstream sector dominates and acts as leader. In this case, an alternative Stackelberg solution is reached, as shown in Table 3, with the supply chain ending in a state of deficit in upstream production capacity. Note that in this case, both controllers incur lower cumulative costs than they do in either of the two previous scenarios. Thus, from the system-level standpoint, this solution is superior for the given transitions and payoffs.

For a closed loop structure the sectors have 16 decision choices that depend on time and the state, see Simaan and Cruz (1973b). In particular, Simaan and Cruz (1973b) showed through the examples that the leader solutions violate Bellman's principle of optimality (Bellman, 1957). In economics, this violation is known as time-inconsistency (Kydland and Prescott 1977). 


\section{CONCLUSIONS}

In this position paper we provide a discussion of the need to use modelling and control methods that are more appropriate than those for single controller systems when we have a networked system of control systems whereby the individual systems that are networked have their individual controls and individual objective functions. This need arises because the network that may involve communication links induces interaction and complicates the choice of control strategies for the various subsystems. There are methodologies that could be applied now for multi-agent systems but there remains further need for research to address issues such as estimation, adaptation, stability, and robustness, to name a few. Global complex systems such as reduction of the external costs of negative environmental impacts of the use of various energy sources, mitigation of natural disasters, and consideration of global warming in technological planning, are prime areas where these networked control systems methods could be beneficially applied.

\section{REFERENCES}

Aviso, K., Tan, R. R., Culaba, A. B., and Cruz, J. B., Jr., 2010, Bi-Level Fuzzy Optimization Approach for Water Exchange in Eco-Industrial Parks, Process Safety and Environmental Protection, Vol. 88, pp. 3140.

Basar, Tamer and Cruz, Jose B., Jr., 1982, Concepts and Methods in Multi-Person Coordination and Control, Optimization and Control of Dynamic Operational Research Models, Ed. S.G.Tzafestas. Amsterdam: North-Holland, 455-462.

Basar, T. and Olsder, G. J., 1998, Dynamic Noncooperative Game Theory. New York, NY: Academic Press, revised ed.

Basar, T. and Selbuz, H., 1979, Closed-loop Stackelberg strategies with applications in the optimal control of multilevel systems, IEEE Trans. on Automatic Control, vol. AC-24, no. 2, pp. 166-179.

Bellman, R., 1957, Dynamic Programming. Princeton, NJ: Princeton University Press.

Castastanon, D. and Athans, M., 1976, On stochastic dynamic Stackelberg strategies, Automatica, vol. 12, pp. 177-183.

Chen, C. I. and Cruz, J. B., Jr., 1972, Stackelberg solution for two person games with biased information patterns, IEEE Trans. on Automatic Control, vol. AC17, pp. 791-797.

Cruz, J. B., Jr., Survey of Nash and Stackelberg
Equilibrium Strategies in Dynamic Games, Annals of Economic and Social Measurement, Vol. 4, No. 4, 1975, pp. 339-344.

Cruz, J. B., Jr., 1978, Leader-follower strategies for multilevel systems, IEEE Trans. on Automatic Control, vol. AC-23, no. 2, pp. 244-255.

Cruz, J. B., Jr., Tan, R. R. Culaba, A. B., and Ballacillo, J.A., 2009, A Dynamic Input-Output Model for Nascent Bioenergy Supply Chains, Applied Energy, Vol.86, Supplement 1, pp. 86-94.

Cruz, Jose B. Jr. and Tan, Xiaohuan, 2009, Dynamic Noncooperative Game Models for Deregulated Electricity Markets, Nova Publishers, New York NY.

Isaacs, R. P., 1955, Differential Games: a Mathematical Theory with Applications to Warfare and Pursuit, Control and Optimization. New York: John Wiley and Sons, 1955.

Kydland, F. E. and Prescott, Edward C., Rules Rather Than Discretion: The Inconsistency of Optimal Plans, The Journal of Political Economy, Vol. 85 No. 3 (June 1977), pp. 473-492.

Nash, J., 1950 Equilibrium points in N-person games, in Proc. of the National Academy of Sciences of the United States of America, vol. 36, pp. 48-49.

Nash, J., 1951, Noncooperative games, Annals of Mathematics, vol. 54, pp. 286-295.

Pontryagin, L. S., Boltyanskii, V. G., Gamkrelidze, R. V.,and Mishchenko, E. F., 1962, The Mathematical Theory of Optimal Processes. New York, NY: Interscience Publishers, 1962.

Simaan, M. and Cruz, J. B., Jr., 1973, On the Stackelberg strategy in nonzero-sum games, Journal of Optimization Theory and Application, vol. 11, no. 5, pp. 533-555.

Simaan, M. and Cruz, J. B., Jr., 1973, Additional aspects of the Stackelberg strategy in nonzero-sum games, Journal of Optimization Theory and Application, vol. 11 , no. 6, pp. 613-626.

Simaan, M. and Cruz, J. B., Jr., 1973, A Stackelberg solution for games with many players, IEEE Trans. on Automatic Control, vol. AC-18, no. 3, pp. 322-324.

Starr, A. W. and Ho, Y. C., 1969, Nonzero Sum Differential Games, Journal of Optimization Theory and Applications, Vol. 3, No. 3.

Starr, A. W. and Ho, Y. C., 1969, Further Properties of Nonzero Sum Differential Games, Journal of Optimization Theory and Applications, Vol. 3, No. 4.

von Neumann, J. and Morgenstern, O., 1947, Theory of Games and Economic Behavior. Princeton, NJ: Princeton University Press, 2nd ed.

von Stackelberg, H., 1952, The Theory of the Market Economy. Oxford: Oxford University Press, English translated ed., 1952.

Wang, Fei-yue and Liu, Derong, 2008, Networked Control Systems: Theory and Applications, Springer. 